\title{
Estimation of umbilical cord blood leptin and insulin based on anthropometric data by means of artificial neural network approach: identifying key maternal and neonatal factors
}

José Guzmán-Bárcenas', José Alfredo Hernández², Joel Arias-Martínez³ , Héctor Baptista-González', Guillermo Ceballos-Reyes ${ }^{4}$ and Claudine Irles ${ }^{1 *}$

\begin{abstract}
Background: Leptin and insulin levels are key factors regulating fetal and neonatal energy homeostasis, development and growth. Both biomarkers are used as predictors of weight gain and obesity during infancy. There are currently no prediction algorithms for cord blood (UCB) hormone levels using Artificial Neural Networks (ANN) that have been directly trained with anthropometric maternal and neonatal data, from neonates exposed to distinct metabolic environments during pregnancy (obese with or without gestational diabetes mellitus or lean women). The aims were: 1) to develop ANN models that simulate leptin and insulin concentrations in UCB based on maternal and neonatal data (ANN perinatal model) or from only maternal data during early gestation (ANN prenatal model); 2) To evaluate the biological relevance of each parameter (maternal and neonatal anthropometric variables).

Methods: We collected maternal and neonatal anthropometric data $(n=49)$ in normoglycemic healthy lean, obese or obese with gestational diabetes mellitus women, as well as determined UCB leptin and insulin concentrations by ELISA. The ANN perinatal model consisted of an input layer of 12 variables (maternal and neonatal anthropometric and biochemical data from early gestation and at term) while the ANN prenatal model used only 6 variables (maternal anthropometric from early gestation) in the input layer. For both networks, the output layer contained 1 variable to UCB leptin or to UCB insulin concentration.

Results: The best architectures for the ANN perinatal models estimating leptin and insulin were 12-5-1 while for the ANN prenatal models, 6-5-1 and 6-4-1 were found for leptin and insulin, respectively. ANN models presented an excellent agreement between experimental and simulated values. Interestingly, the use of only prenatal maternal anthropometric data was sufficient to estimate UCB leptin and insulin values. Maternal BMI, weight and age as well as neonatal birth were the most influential parameters for leptin while maternal morbidity was the most significant factor for insulin prediction.

(Continued on next page)
\end{abstract}

\footnotetext{
*Correspondence: cloirles@gmail.com; claudine.irles@inper.gob.mx

'Department of Physiology and Cellular Development, Instituto Nacional de

Perinatología Isidro Espinoza de los Reyes (INPerlER), Montes Urales 800,

Lomas de Virreyes, Mexico city C.P. 11000, Mexico

Full list of author information is available at the end of the article
} 
(Continued from previous page)

Conclusions: Low error percentage and short computing time makes these ANN models interesting in a translational research setting, to be applied for the prediction of neonatal leptin and insulin values from maternal anthropometric data, and possibly the on-line estimation during pregnancy.

Keywords: Mathematical model, Leptin, Insulin, Neonate, Artificial neural network, Umbilical cord blood, Gestational diabetes, Maternal obesity

\section{Background}

The impact of maternal obesity during pregnancy (maternal overweight/obesity with or without gestational diabetes mellitus) and its association with an increased risk of obesity, as well as re-programming cardiovascular risk, body composition and cardiometabolic health in infancy and early adulthood, has been shown in humans and animal models [1-7]. It has been demonstrated that human obesity in children and adults is associated with elevated serum levels of an adipokine, the hormone leptin, reflecting the amount of energy stored in adipose tissue $[8,9]$. Leptin was identified as the product of the obesity $(o b)$ gene [10], which is secreted into the circulation by large adipocytes, and has been shown to cross the blood-brain barrier and bind to specific receptors in the hypothalamus to alter the expression of several neuropeptides that regulate neuroendocrine function, energy intake and expenditure leading to a decrease in appetite, reduction of body fat and body weight $[11,12]$. The pancreatic hormone insulin also acts in the brain as a negative feedback signal for adipocity. It is also an essential regulator of growth, increasing fat deposition [13] resulting in a greater potential for leptin synthesis by stimulating adipocyte $o b$ gene transcription [14]. As well, leptin also modulates (increases) insulin secretion by pancreatic $\beta$ cells [15]. Therefore, leptin and insulin control glucose metabolism, acting at the peripheral and central level [16]. During pregnancy, leptin levels regulate fetal development and growth $[17,18]$ and positively correlate in umbilical cord blood (UCB) with neonatal body weight and fat mass [9]. It has been demonstrated that UCB leptin concentration correlates with insulin levels and anthropometric data (birth weight) only in large for gestational age neonates, but they do not correlate with maternal levels [19-21]. Both leptin and insulin biomarkers are used as predictors of weight gain and obesity during infancy. Indeed, several studies have shown that lower cord blood leptin levels predict an increased weight and length gain, "catch-up" growth, as well as a higher BMI in infancy ( $2-3$ years) [22$24]$. Yet, in the first months, decreased cord leptin levels together with gestational diabetes mellitus are related to a slower weight gain [25]. For insulin, an inversely relationship was found for weight gain during infancy [26]. Therefore, the prediction of cord blood hormone levels based on anthropometric maternal and neonatal data using mathematical models that take into account the high complexity of this system may be of considerable usefulness. Therefore, Artificial Neural Networks (ANNs) will be used as a system biology approach to simulate cord blood hormone levels.

ANNs [27] have been extensively used for the optimization and modeling of processes, as they are able to represent the non-linear dynamic interaction of complex relationships without any assumptions of the underlying mechanisms [28]. ANNs learn and test the solution of the problem from a data set [29] and provide an interpolation for new data. For the science of medicine, the application of neural networks keeps on expanding [30-32], and now represents a set of methods that have been useful for solving pediatric problems [33, 34], identifying key factors such as in fetal growth $[35,36]$ and diagnosing neonatal diseases [37].

ANN has the ability to predict data such as measuring biochemical parameters in UCB samples which may be difficult to obtain otherwise. The objectives of the study were therefore: 1 ) to obtain ANN models (feed-forward) for the prediction of leptin and insulin values in UCB from neonates exposed to distinct metabolic environments during pregnancy (defined as obesity with or without gestational diabetes mellitus or lean women), based on anthropometric maternal and neonatal characteristics (ANN perinatal model) or from only maternal data during early gestation (ANN prenatal model) 2), to examine which parameters, among those analyzed from the mother and neonate, have the most influence on neonatal leptin and insulin values by applying a sensitivity analysis. Essentially, ANN will learn from a database (maternal and neonatal clinical data as well as biochemical experimental data) from a specified problem (maternal metabolic environment) with a known solution (UCB leptin and insulin experimental values for training the model) and then the network, will recreate the system of an inherent complex set of data (testing the model). 


\section{Methods}

\section{Study subjects}

This study was approved by both the Ethics and Research Committees of the Instituto Nacional de Perinatologia "Isidro Espinosa de los Reyes". Venous umbilical cord blood samples were collected from 49 cesarean deliveries. Samples were centrifuged (for $15 \mathrm{~min}$, at $3500 \mathrm{rpm}$, room temperature), serum was aliquoted and stored at $-70{ }^{\circ} \mathrm{C}$ until assayed. Hemolyzed or lipemic samples were discarded. Mothers $(N=49)$ were: lean normoglycemic (initial Body Mass Index, BMI, of $24.3 \pm$ $0.4 \mathrm{~kg} / \mathrm{m}^{2}, n=11$ ), obese (initial BMI of $30.9 \pm 0.9 \mathrm{~kg} / \mathrm{m}^{2}$, $n=23$ ) and obese with gestational diabetes mellitus (initial BMI of $31.3 \pm 0.7 \mathrm{~kg} / \mathrm{m}^{2}, n=15$ ). We collected the following information: maternal morbidity (MM), gestational age at delivery (GE), initial and final maternal weight (MWi and MWf), initial and final BMI (MBMi and MBMf), maternal height $(\mathrm{MH})$, maternal age (MA), parity $(\mathrm{P})$, neonatal gender (NG), neonatal birth weight $(\mathrm{NW})$, neonatal body length $(\mathrm{NH})$, neonatal head circumference (NHC), neonatal BMI (NBMI) and 5-min APGAR score. The age range of the participants was 16-43 years. Exclusion criteria were genetic syndromes, chromosomal abnormalities, gross placental abnormalities, infections and substance abuse. The main clinical data are reported in Table 1.

Table 1 Maternal and neonatal clinical data

\begin{tabular}{|c|c|c|c|}
\hline \multirow[b]{2}{*}{ Parameters } & \multirow[b]{2}{*}{ Healthy } & \multicolumn{2}{|l|}{ Mother } \\
\hline & & Obese & Diabetic \\
\hline$N=49$ & 11 & 23 & 15 \\
\hline Maternal age (years) & $25.1( \pm 3.3)$ & $30.3( \pm 1.1)$ & $35( \pm 1.2)$ \\
\hline Maternal initial weight (kg) & $58.3( \pm 2.1)$ & $74.2( \pm 2.5)$ & $77.3( \pm 2.7)$ \\
\hline Maternal final weight (kg) & $67.9( \pm 2.6)$ & $85.6( \pm 3.2)$ & $89.4( \pm 3.9)$ \\
\hline Maternal height (cm) & $\begin{array}{l}156.4 \\
( \pm 2.2)\end{array}$ & $\begin{array}{l}157.1 \\
( \pm 1.3)\end{array}$ & $157.3( \pm 1)$ \\
\hline $\begin{array}{l}\text { Gestational age at delivery, } \\
\text { (weeks) }\end{array}$ & $38.8( \pm 0.2)$ & $38( \pm 0.4)$ & $39( \pm 0.3)$ \\
\hline Initial Maternal BMI (kg/m²) & $24.3( \pm 0.4)$ & $30.7( \pm 0.8)$ & $31.3( \pm 0.8)$ \\
\hline Final Maternal BMI $\left(\mathrm{kg} / \mathrm{m}^{2}\right)$ & $27.7( \pm 0.5)$ & $34.7( \pm 1.2)$ & $36( \pm 1.4)$ \\
\hline Parity & $1.7( \pm 0.3)$ & $2.7( \pm 0.2)$ & $2.6( \pm 0.3)$ \\
\hline Males/Females & $5 \mathrm{M} / 6 \mathrm{~F}$ & $7 \mathrm{M} / 8 \mathrm{~F}$ & $11 \mathrm{M} / 11 \mathrm{~F}$ \\
\hline Neonatal birth weight (kg) & $\begin{array}{l}2.87 \\
( \pm 0.14)\end{array}$ & $\begin{array}{l}2.88 \\
( \pm 0.09)\end{array}$ & $\begin{array}{l}3.22 \\
( \pm 0.09)\end{array}$ \\
\hline Neonatal birth body length $(\mathrm{cm})$ & $47.9( \pm 0.6)$ & $47.9( \pm 0.4)$ & $48.3( \pm 0.7)$ \\
\hline $\begin{array}{l}\text { Neonatal head circumference } \\
(\mathrm{cm})\end{array}$ & $34.4( \pm 0.3)$ & $33.8( \pm 0.2)$ & $34.7( \pm 0.3)$ \\
\hline Neonatal BMI & $\begin{array}{l}12.45 \\
( \pm 0.4)\end{array}$ & $\begin{array}{l}12.52 \\
( \pm 0.3)\end{array}$ & $\begin{array}{l}13.88 \\
( \pm 0.4)\end{array}$ \\
\hline 5-min APGAR score & 9 & $8.9( \pm 0.07)$ & $9( \pm 0.06)$ \\
\hline
\end{tabular}

All values are depicted as Mean +/- SEM
Leptin and Insulin determination by ELISA

Leptin and insulin from umbilical cord blood samples were assayed using commercially available ELISA kits (GenWay, San Diego, CA). The sensitivity of the leptin assay was $0.1 \mathrm{ng} / \mathrm{ml}$; intra-assay and inter-assay coefficients of variations were 4.2 and $6.7 \%$, respectively. The sensitivity of the insulin assay was $0.3 \mu \mathrm{U} / \mathrm{ml}$; intra-assay and inter-assay coefficients of variations were 6.3 and $8.5 \%$, respectively.

\section{Database}

We compared neonates and their mothers with distinct metabolic environments during pregnancy (defined as obese, obese with gestational diabetes mellitus or lean women). Fourteen variables were selected from the entire database for 49 subjects. For the ANN models, anthropometric, maternal morbidity (obese, obese with gestational diabetes mellitus or lean women) and biochemical data (umbilical cord blood leptin or insulin) were administered.

\section{ANN learning and testing}

Back-propagation (BP) algorithm multiple-layer perceptron (MLP) architecture was trained and tested by the input layer, the hidden layer and the output layer (see Fig. 1). We applied the Log-sigmoid (LOGSIG) and hyperbolic tangential (TANSIG) transfer functions in the hidden layer. Both transfer functions were acceptable however; the hyperbolic tangential performance was slightly superior. According to [38], TANSIG transfer function has a better performance which is in agreement with our result. In the output layer, only the linear transfer function (PURELIN) was employed because the output layer is not normalized. In order to obtain the optimum model, we began in the hidden layer with one neuron until the Root Mean Square Error (RMSE) did not change and the statistical test (slope and intercept [39]) was approved, as well as we avoided over-fitting (for a detailed explanation see Additional file 1) and [40-43]. All calculations were carried out with Matlab mathematical software (Natick, MS, USA) with the Neural Network Toolbox for Matlab [40].

The input layer for the ANN perinatal models consisted of 12 maternal and neonatal variables and the output layer contained one variable for umbilical cord blood leptin or umbilical cord blood insulin concentrations. The variables were: maternal morbidity (MM), gestational age at delivery (GE), initial and final maternal weight (MWi and MWf), initial and final maternal BMI (MBMi and MBMf), maternal height $(\mathrm{MH})$, neonatal gender $(\mathrm{NG})$, neonatal birth weight $(\mathrm{NW})$, neonatal body length $(\mathrm{NH})$, neonatal head circumference (NHC) and neonatal BMI (NBMI) (Table 2). The input layer for the ANN prenatal models (early gestation) contained 6 maternal variables (maternal morbidity 

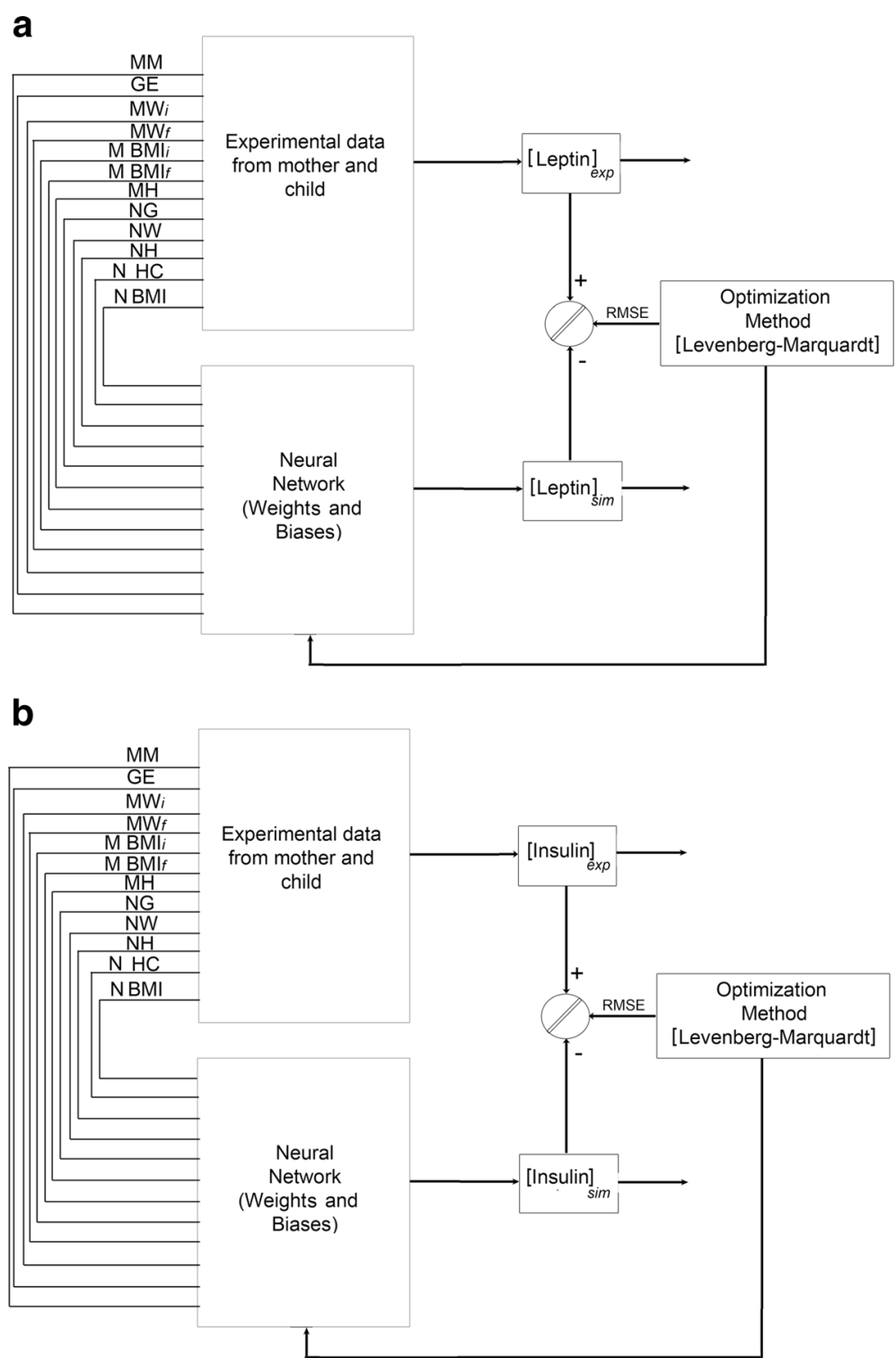

Fig. 1 Recurrent network architecture of the ANN perinatal model and the procedure used for learning neural network for the simulation of leptin (a) and insulin (b) concentration in umbilical cord blood (UCB) samples

(MM), initial maternal weight (MWi), initial maternal BMI (MBMi), maternal height (MH), maternal age (MA) and parity, P) and the output layer had one variable for umbilical cord blood leptin or umbilical cord blood insulin concentrations (Table 2).

In the learning, to change the weights and biases, we applied the Levenberg-Marquardt (LM) algorithm as the learning (training) algorithm allowing to obtain a smaller RMSE [40, 44, 45] (for a detailed explanation see Additional file 1). The RMSE was calculated from the experimental values and network predictions (see Fig. 1a and $b$ ).

The experimental database $(n=49)$ was used to feed the ANN structure. This database $\left(x_{i}\right)$ was randomly divided into: learning (79 \%) and validation (21\%). The database was then normalized in the range of 0.1 to 0.9 [46] for the input variables and the output variable was not normalized.

So, the entire input database was scaled to a new value $x_{i}$ as follows:

$$
x_{i}=0.8\left(\frac{X_{i}-X_{\min }}{X_{\max }-X_{\min }}\right)+0.1
$$

\section{Statistical test}

In order to confirm the best performance of the ANN predictions, a linear regression was carried out to obtain 
Table 2 List of experimental variables (clinical and biochemical data) analyzed using ANN to obtain umbilical cord blood leptin and insulin values: input and output range conditions studied

\begin{tabular}{|c|c|c|c|}
\hline Input Variables $(n=49)$ & Range & Output variables & Range \\
\hline Maternal Morbidity, MM & Healthy, Obese or diabetic & \multirow{2}{*}{$\begin{array}{l}\text { Umbilical cord } \\
\text { blood leptin, (ng/ml) }\end{array}$} & \multirow[t]{2}{*}{$0.17-27$ (mean 5.1) } \\
\hline Maternal initial weight, MWi (kg) & 49-96 (mean 72) & & \\
\hline Maternal final weight, MWf (kg) & 55-117 (mean 83) & $\begin{array}{l}\text { Umbilical cord } \\
\text { blood insulin, }(\mu \mathrm{U} / \mathrm{ml})\end{array}$ & $0.7-12$ (mean 1.9) \\
\hline Maternal height, MH (cm) & 149-173 (mean 157) & & \\
\hline Maternal initial BMI, MBMi $\left(\mathrm{kg} / \mathrm{m}^{2}\right)$ & 22-40 (mean 29.5) & & \\
\hline Maternal final BMI, MBMf $\left(\mathrm{kg} / \mathrm{m}^{2}\right)$ & 24-42 (mean 33.5) & & \\
\hline $\begin{array}{l}\text { Gestational age at delivery, GE (weeks) } \\
\text { Neonatal gender, NG }\end{array}$ & 37-41 (mean 39) & & \\
\hline Neonatal birth weight, NW (kg) & 2.01-4.19 (mean 2.98) & & \\
\hline Neonatal birth body length, $\mathrm{NH}(\mathrm{cm})$ & 45-54 (mean 48) & & \\
\hline Neonatal head circumference, NHC (cm) & 32-37 (mean 34) & & \\
\hline Neonatal BMI, NBMI & 10-15 (mean 13) & & \\
\hline Parity, P & 1-5 (mean 2.3) & & \\
\hline Maternal age, MA (years) & 16-43 (mean 30) & & \\
\hline
\end{tabular}

the slope and intercept from the ANN simulations versus the experimental database (learning and validation database), after which we applied a statistical test (slope and intercept, [39, 47]). This last consists in demonstrating that the obtained upper and lower intervals of the slope must be one and the upper and lower intervals of the intercept must be zero, with a $99.8 \%$ confidence level according to the Student t-test.

\section{Results}

The main umbilical cord blood leptin and insulin experimental values are reported in Table 3 and are in agreement with the study by [48] (as well as [49-51]). Briefly, obesity exposed neonates had higher UCB leptin levels compared to not exposed neonates and a trend for increased levels in gestational diabetes exposed neonates. Gestational diabetes exposed neonates had the highest values for umbilical cord blood insulin levels than not exposed or obesity exposed neonates.

\section{Proposed ANN perinatal model}

The input variables for the ANN perinatal models were 12 (maternal and neonatal) and were presented to the general network, in which the final UCB hormone level prediction corresponded to the output unit: 1 output variable for UCB leptin or insulin concentration. Figure 2 shows the general scheme of such neural network architecture for the prediction of UCB leptin (Fig. 2a) and insulin (Fig. 2b) values from perinatal parameters (as depicted in Fig. 1a and b). 20,000 runs with 100 iterations were applied in each neuron from 1 to 5 neurons in the hidden layer and the final topology was obtained for leptin and insulin predictions. As a result, the best network architecture performance was 12-5-1 for both models: leptin and insulin (Equations [6-11] and the weights and biases are reported in Additional file 1: Tables S1 and S2).

\section{Validation of the ANN perinatal model}

Figure 3 depicts the comparison of the experimentally measured (EXP) and the predicted (ANN) UCB leptin (Fig. 3a) and insulin (Fig. 3b) values for the testing database describing the behavior of the ANN perinatal model using all data available (inputs).

The comparison of (EXP) and (ANN) data through a linear regression model,

$\left(\right.$ Leptin $_{A N N}=a+b$ Leptin $\left._{E X P}\right)$ and $\left(\right.$ Insulin $_{A N N}=a+b$ Insulin $_{E X P}$ ), showed regression coefficients of $R^{2}>0.973$ and $R^{2}>0.9873$ for leptin and insulin, respectively. Upper and lower values of the statistical test (Table 4) indicate that the slope included one and the intercept contained zero, with a $99.8 \%$ confidence level for both UCB determinations [39, 47]. These results demonstrated a good correlation between ANN predictions and experimental values.

Table 3 Umbilical cord blood hormone concentrations

\begin{tabular}{llll}
\hline & & Mother & \\
Parameters & Healthy & Obese & Diabetic \\
\hline$N=49$ & 11 & 23 & 15 \\
Leptin $(\mathrm{ng} / \mathrm{ml})$ & $3.5( \pm 1)$ & $6.7( \pm 1.5)$ & $4.9( \pm 0.4)$ \\
Insulin $(\mu \mathrm{U} / \mathrm{ml})$ & $1.3( \pm 0.4)$ & $1.06( \pm 0.08)$ & $3.6( \pm 1)$ \\
\hline
\end{tabular}




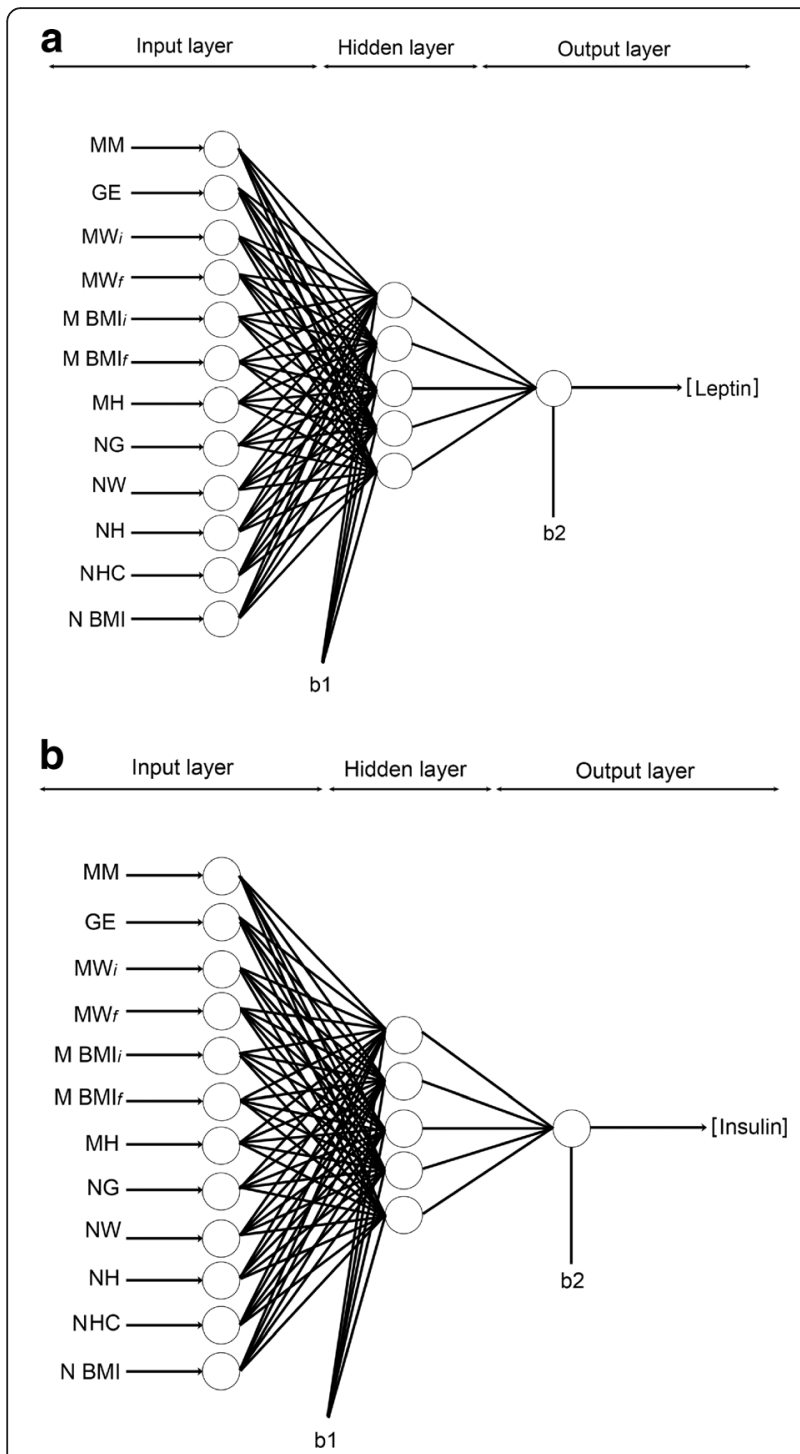

Fig. 2 The neural network computational ANN perinatal model for UCB leptin (a) and insulin (b) concentration estimation. The proposed model involved 12 input variables, 5 neurons on hidden layer and 1 output variable

\section{Sensitivity analysis of the ANN perinatal model}

We used an evaluation process based on the neural network weight matrix and the Garson equation $[52,53]$ to obtain the qualitative significance of the input variables on the predicted UCB leptin and insulin values (for a detailed explanation of Equation [19], see Additional file 1 ). Figure 4 depicts the relative importance of the calculated input variables showing that all variables had a strong effect on leptin (Fig. 4a) and insulin (Fig. 4b) neonatal values. In addition, the sensitivity analysis showed that maternal BMI (28\%, initial and final BMI), neonatal birth weight $(12 \%)$ and maternal weight $(11 \%)$ were the most influential factors controlling umbilical cord blood leptin concentration, in contrast with maternal

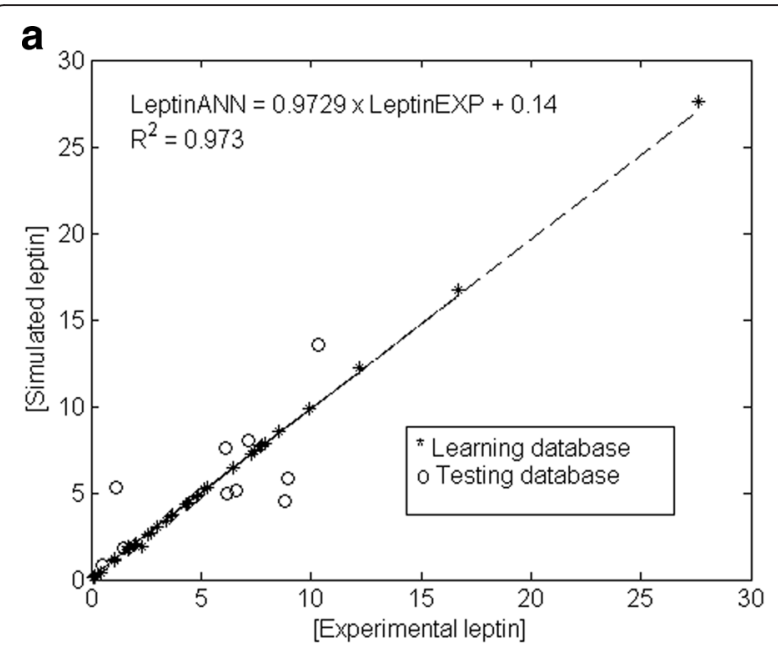

b

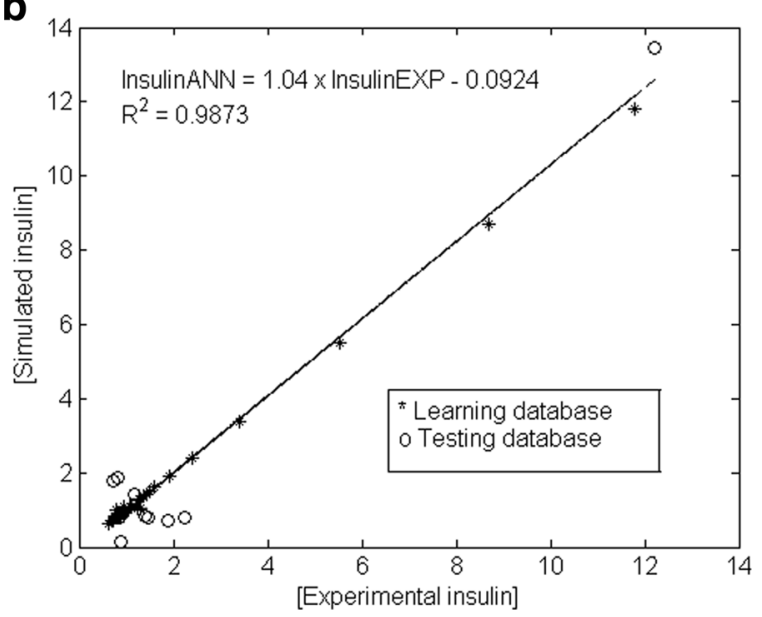

Fig. 3 The scatter plot of perinatal experimental (open circles) vs. ANN-predicted values (dark cross) for average UCB leptin (a) and insulin (b). Experimental (leptin EXP and insulin $n_{\text {EXP }}$ ) and simulated (leptin $_{\text {ANN }}$ and insulin ${ }_{\text {ANN }}$ ) data. Dashed line indicates the fitted simple regression line on scattered points

morbidity (healthy, obese or controlled gestational diabetes, $5 \%)$ and neonatal BMI (2\%) that were the less important factors for estimating UCB leptin levels (Fig. 4a).

For UCB insulin concentration, maternal morbidity (healthy, obese or controlled gestational diabetes) appears to be the critical parameter with a relative importance of $18 \%$, followed by neonatal body length at

Table 4 Intercept (a) and slope (b) statistical test to leptin and insulin in the ANN perinatal model

\begin{tabular}{llll}
\hline Leptin & & Insulin & \\
\hline$a_{\text {lower }}$ & $a_{\text {upper }}$ & $a_{\text {lower }}$ & $a_{\text {upper }}$ \\
-0.6321 & 0.9118 & -0.3411 & 0.1564 \\
$b_{\text {lower }}$ & $b_{\text {upper }}$ & $b_{\text {lower }}$ & $b_{\text {upper }}$ \\
0.8624 & 1.0833 & 0.9564 & 1.1156 \\
\hline
\end{tabular}


delivery (12 \%), maternal BMI (6-9 \%) and less importantly neonatal BMI (5\%) and birth weight (5\%) (Fig. 4b).

Altogether, these results showed that the ANN perinatal models succeeded in predicting the experimental results of UCB leptin and insulin concentration from anthropometric maternal and neonatal values, as well as revealed a good agreement between the experimental data and the predicted values. However, this ANN perinatal models required gestation and at term information in order to predict UCB leptin and insulin concentrations. Therefore, a second ANN model was applied to predict UCB levels using only inputs from early gestation of the same database.

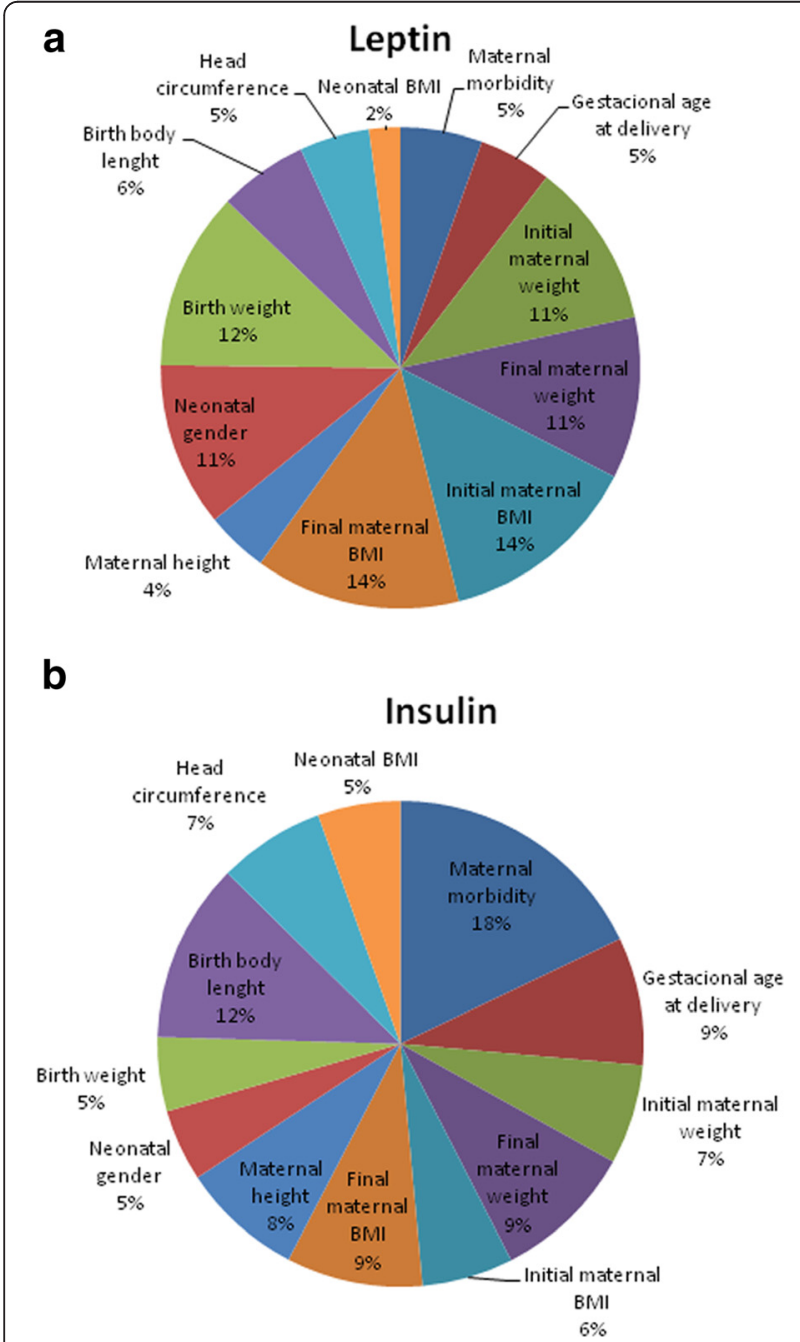

Fig. 4 Percentage for the global sensitivity analysis of the 12 input variables in the ANN perinatal model for UCB leptin (a) and Insulin (b) values

\section{Proposed ANN prenatal model}

The input variables for the ANN prenatal models (early gestation) were 6 maternal and 1 output variable for UCB leptin or insulin concentration. The best neural network architecture obtained for the prediction of UCB leptin was 6-5-1 (Fig. 5a) and for UCB insulin, 6-4-1 (Fig. 5b) (The same Equations [6-11 for leptin and 6-10 for insulin] were utilized in the model but the weights and biases for leptin and insulin simulations are reported in Additional file 1: Tables S3 and S4). The ANN models were able to predict UCB leptin and insulin levels from only anthropometric maternal parameters.

\section{Validation of the ANN prenatal model}

Figure 6 depicts the predicted values compared to the experimental values for leptin (Fig. 6a) and insulin (Fig. 6b), showing a good capability of the model to simulate both outputs by describing the behavior of the UCB levels using only maternal anthropometric information from early gestation.

The regression coefficients were $R^{2}>0.963$ and $R^{2}>$ 0.9824 for leptin and insulin, respectively (Fig. 6). Table 5 depicts intercepts and slopes for the linear regression model of the ANN prenatal models. Therefore, these

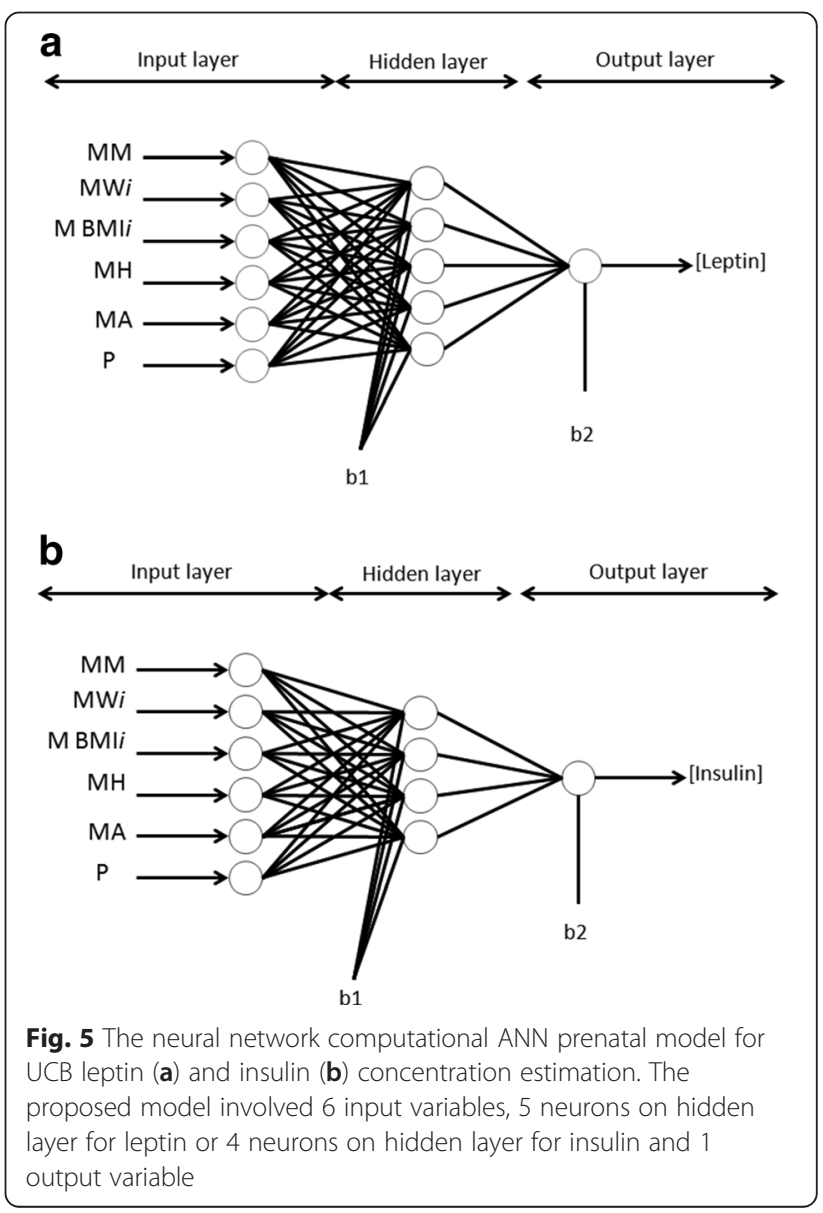


statistical results guarantee the validity of the ANN prenatal models with a confidence level of $99.8 \%$ for both UCB leptin and insulin $[39,47]$.

\section{Sensitivity analysis of the ANN prenatal model}

The same process used for the ANN perinatal models allowed obtaining the relative importance of the input variables on the simulated UCB leptin (Fig. 7a) and insulin (Fig. 7b) values in the ANN prenatal models. Figure $7 \mathrm{a}$ shows that maternal age $(27 \%)$ and initial maternal weight $(24 \%)$ were the dominant factors for the prediction of UCB leptin in comparison with maternal morbidity (11\%) and parity $(8 \%)$, which were the less important parameters. For UCB insulin simulation (Fig. 7b), all maternal characteristics had a strong effect on insulin values but maternal morbidity (31 \%) and maternal height $(25 \%)$ were the predominant parameters followed by maternal age $(11 \%)$ and parity $(10 \%)$.

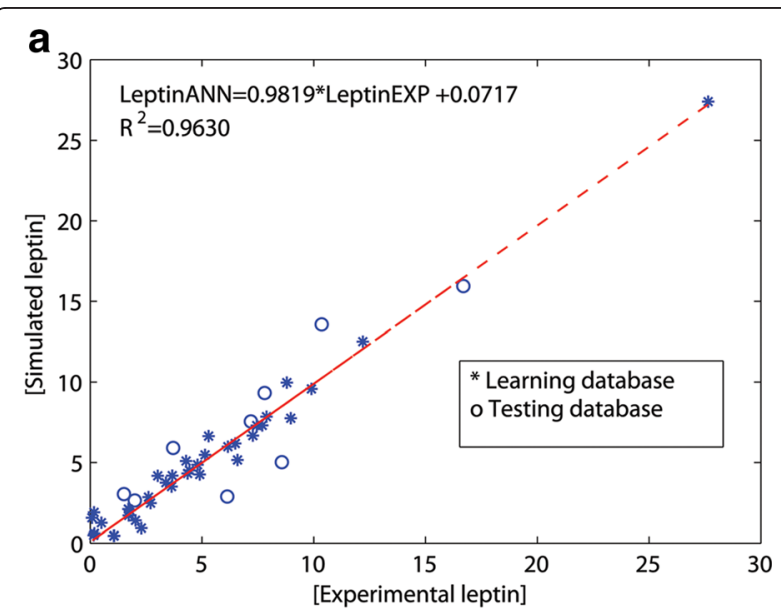

\section{b}

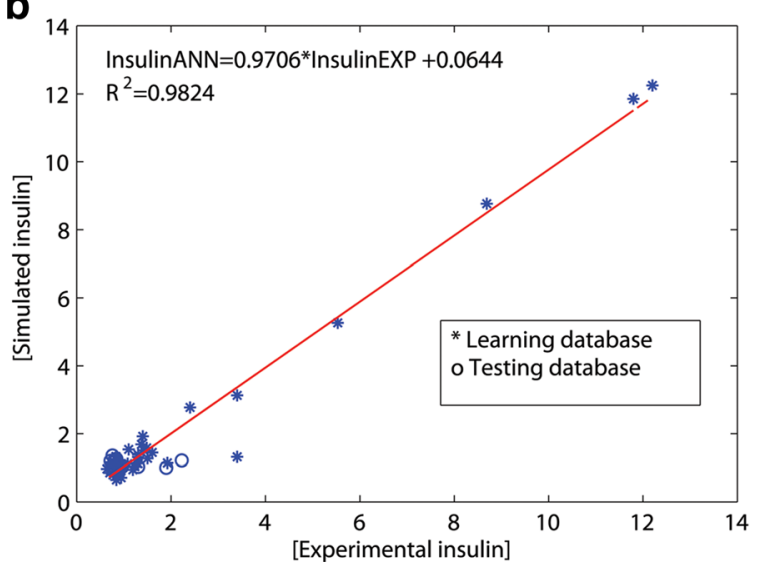

Fig. 6 The scatter plot of prenatal experimental (open circles) vs. ANN-predicted values (dark cross) for average UCB leptin (a) and insulin (b). Experimental (leptin Exp and insulin Exp) and simulated (leptin ANN and insulin ANN) data. Dashed line indicates the fitted simple regression line on scattered points
Table 5 Intercept (a) and slope (b) statistical test to leptin and insulin in the ANN prenatal model

\begin{tabular}{llll}
\hline Leptin & & Insulin & \\
\hline$a_{\text {lower }}$ & $a_{\text {upper }}$ & $a_{\text {lower }}$ & $a_{\text {upper }}$ \\
0.8508 & 1.1131 & 0.8838 & 1.0574 \\
$b_{\text {lower }}$ & $b_{\text {upper }}$ & $b_{\text {lower }}$ & $b_{\text {upper }}$ \\
-0.8453 & 0.9886 & -0.2069 & 0.3357 \\
\hline
\end{tabular}

\section{Discussion}

Four ANNs models were developed to predict neonatal leptin and insulin concentrations in umbilical cord blood, based on selected experimental conditions (anthropometric and biochemical variables), from gestation and at term data (ANN perinatal models) or only from early gestation data (ANN prenatal models). The neonatal leptin and insulin parameters were successfully simulated by applying in all models, a three layered neural network with 4-5 neurons in the hidden layer, using a back-propagation algorithm that achieved a low average error rate $(<3$ and $<4 \%$, for the ANN perinatal

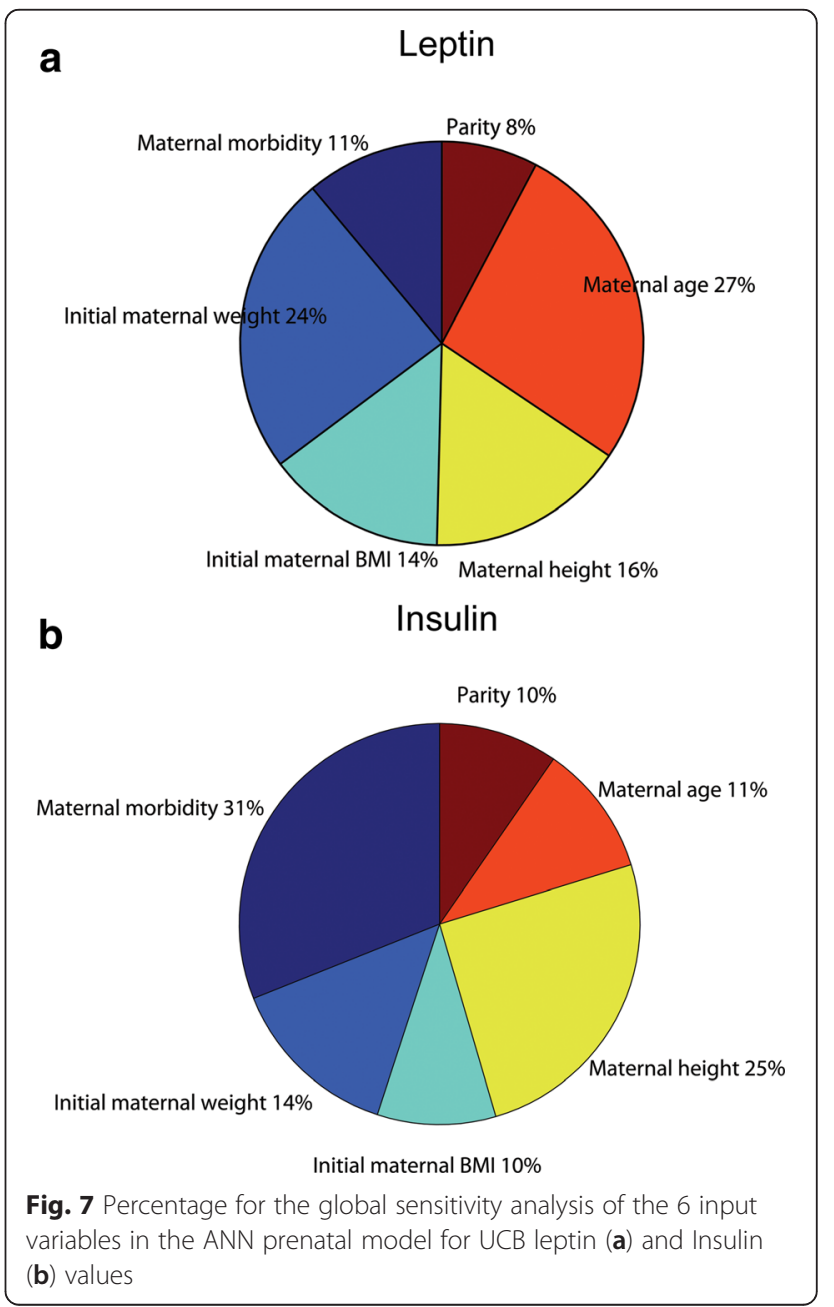


models and ANN prenatal models, respectively). The results obtained by the ANN perinatal models show a high agreement with experimental results: a good correlation $\left(R^{2}>0.97\right)$ and small error $(R M S E>0.0012)$. High level of confidence for the ANN perinatal models was confirmed with the intercept and slope statistical test (99 \%). Interestingly, the ANN prenatal models, that takes into account only the early gestation information (maternal anthropometric parameters), were also able to estimate leptin and insulin values with a good correlation coefficient $\left(R^{2}>0.96\right.$ for leptin and $R^{2}>0.98$ for insulin), a small error $(R M S E>0.2)$ and a confidence level of $99.8 \%$. These models consider well-known and simple to measure input parameters such as: corporal weight, length and body mass index of the mother at the beginning and/or end of pregnancy, gestational age at delivery, gender, weight, body length and head circumference of the neonate at delivery. Therefore, by means of these ANN models we could be able to obtain any unknown leptin and insulin variables based exclusively in anthropometric data.

ANN was, not only capable of establishing mathematical models estimating neonatal leptin and insulin values in umbilical cord blood from anthropometric values, but was also able to identify key maternal and neonatal variables, which had mathematically consistent biological relevance for the predicted values. According to the sensitivity analysis of the ANN perinatal models, we found that maternal BMI and neonatal birth weight were the most influential parameters for the prediction of neonatal leptin values, while maternal metabolic health was the principal factor for the simulation of neonatal insulin levels. Interestingly, the sensibility analysis of the ANN prenatal models (taking into account only early gestation maternal anthropometric values) showed maternal age and initial maternal weight had a strong impact on UCB leptin levels, whereas maternal metabolic health was the most important parameter for fetal insulin secretion.

These analyses were capable of confirming a major role of maternal BMI and birth weight for UCB leptin prediction and maternal metabolic health for insulin values. Indeed, it has been shown that UCB leptin concentration correlates with maternal BMI and neonatal birth weight by conventional observational and statistical methods [18-20, 54, 55]. In particular, maternal BMI as a key factor for UCB leptin levels is in agreement with the proposed mechanism for leptin during pregnancy. In fact, the adipose tissue, the placenta and the vascular endothelium of the mother have been demonstrated as sites for regulated leptin production in utero [56, 57] and during pregnancy, leptin has been shown to regulate protein synthesis, growth and immunity [58].

For UCB insulin values, maternal metabolic health (healthy, obese or obese with gestational diabetes mellitus) appears to be the critical parameter, followed by neonatal body length. Indeed, gestational diabetes mellitus exposed neonates had higher umbilical cord blood insulin levels than not exposed neonates [48, 59], which was confirmed by the ANN analysis. The fetal pancreas is the principal source of fetal insulin since maternal insulin does not cross the blood/placental barrier [60]. The fact that insulin is higher in gestational diabetes mellitus offspring may suggest dysregulation of insulin signaling at birth which is compatible with an adaptation for elevated maternal glucose levels [61]. Prediction of UCB insulin levels by neonatal body length is in agreement to insulin's direct anabolic action. This has been proposed to be indirectly mediated via leptin, since UCB leptin levels strongly correlate with UCB insulin values [21]. Also, it has been hypothesized that fetal insulin stimulates fetal adipocyte leptin production [21]. However, UCB leptin levels did not correlate with UCB insulin levels in the offspring of obese women with gestational diabetes mellitus.

\section{Limitations and strengths of the ANN models}

It is important to acknowledge the limitations of this study such as the particularly low sample size. However, adaptive learning algorithms like ANN were able to overcome this problem of low sample size due to the training procedure that uses only a part of the database. It is noteworthy to mention that a study from Street and cols used a similar size database for their ANN model in order to identify placental factors for fetal growth [36]. Another limitation of this work is the low test data size. Further simulations with an increased sample size should allow improving the ANN models.

In addition, the limits of the modeling are that in order to predict UCB leptin and insulin concentrations, morbidity, anthropometric and biochemical parameters must be placed between the ranges of the input variables (see Table 2). For example, the ANN perinatal model will accurately simulate UCB leptin when applied to mothers with an initial weight $\left(\mathrm{MW}_{i}\right)$ comprised between 49$96 \mathrm{~kg}$ and an initial BMI $\left(\mathrm{MBMI}_{i}\right)$ of $22-40 \mathrm{~kg} / \mathrm{m}^{2}$.

One of the strengths of this ANN approach is that the elapsed time to calculate both neonatal parameters is short which can be applied on-line and that it represents the dynamic interactions of complex relationships. These characteristics suggest a possible translational utility of these ANN models.

Insulin and leptin cord blood levels have been used as predictor of postnatal growth and weight gain in infancy. So, the establishment of ANN prenatal models that predicts these values from maternal anthropometric variables during gestation, without the need for cord blood samples, could be helpful to prognosticate infant growth and permit the possibility of conducting interventions. 
For example, it is tempting to speculate that infants from obese mothers that had the highest cord leptin values will probably have the worst of all weight gain but the highest BMI in infancy, since higher cord blood leptin and gestational diabetes are related to a slower weight gain and an increased BMI at 2-3 years [23, 24]. The use of these ANN models could make easy to follow growth and perhaps estimate the risk of obesity and diabetes in these children.

\section{Conclusions}

Low error percentage and short computing time makes this ANN models attractive to be applied for the prediction of UCB leptin and insulin values from maternal and neonatal anthropometric data, and possibly the on-line estimation during pregnancy, birth and infancy. In particular, the prediction of these hormone values in UCB may be of great interest to prognosticate infant growth and permit the possibility of conducting interventions before incurring costly and time-consuming events, such as neonatal morbidity. Moreover, the fact that the ANN prenatal model, based merely on early gestation anthropometric maternal information, was able to confidently simulate UCB leptin and insulin levels, make these particular models an interesting application for following the impact of maternal anthropometrics and metabolic health on these hormone UCB values and to predict leptin and insulin values at birth from early gestation.

\section{Additional file}

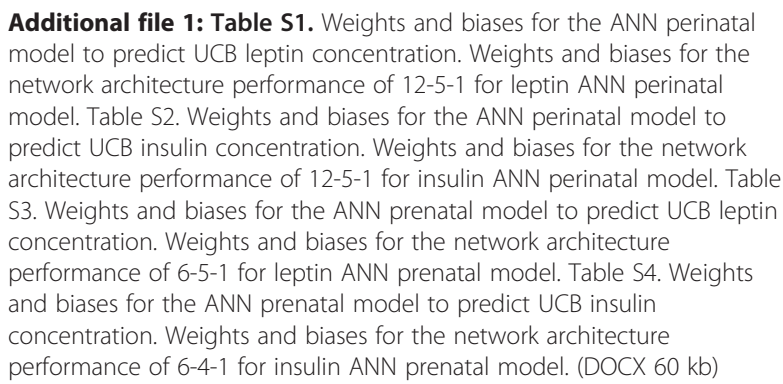

\section{Abbreviations}

ANN, artificial neural network; BP, back-propagation algorithm; b, biases; BMI, body mass index; EXP, experimentally measured; MBMf, final maternal BMl; MWf, final maternal weight; GE, gestational age at delivery; TANSIG, hyperbolic tangential transfer function; MBMi, initial maternal BMI; MWi, initial maternal weight; LM, Levenberg-Marquardt algorithm; LOGSIG, log-sigmoid transfer function; MA, maternal age; $\mathrm{MH}$, maternal height; $\mathrm{MM}$, maternal morbidity; MLP, multiple-layer perceptron; NW, neonatal birth weight; NBMI, neonatal $\mathrm{BMI} ; \mathrm{NH}$, neonatal body length; $\mathrm{NG}$, neonatal gender; $\mathrm{NHC}$, neonatal head circumference; P, parity; ANN, predicted; RMSE, root mean square error; UCB, umbilical cord blood; W, weights.

\section{Acknowledgements}

This work was supported by the Instituto Nacional de Perinatología "sidro Espinoza de los Reyes" and SEP-CONACYT [grant numbers 49350 to Cl]. We also thank for $\mathrm{L}$. Correa for secretarial assistance.

\section{Author disclosure statement}

We declare that there is no conflict of interest, nor commercial association.

Funding

This work was supported by SEP-CONACYT [grant number 49350 to Cl].

\section{Availability of data and materials}

The datasets supporting the conclusions of this article are included within the article (and its additional files).

\section{Authors' contributions}

$J G$ and JA carried out the immunoassays. GC and HB participated in the design of the study. JH performed the ANN approach. JG, $\mathrm{Cl}$ and JH conceived the study, participated in its design, coordination and draft the manuscript. All authors read and approved the final manuscript.

\section{Competing interests}

The authors declare that they have no competing interests.

\section{Consent for publication}

Not applicable.

\section{Ethics approval and consent to participate}

This study involving human participants was approved by the Ethics Committee of the Instituto Nacional de Perinatologia "sidro Espinosa de los Reyes" and was carried out in accordance with the ethical standards of the IRB and the Helsinki Declaration. Written consent for participation was obtained from the subjects or their surrogates (parents or legal tutor) as appropriate.

\section{Author details}

'Department of Physiology and Cellular Development, Instituto Nacional de Perinatología Isidro Espinoza de los Reyes (INPerlER), Montes Urales 800, Lomas de Virreyes, Mexico city C.P. 11000 , Mexico. ${ }^{2}$ Centro de Investigación en Ingeniería y Ciencias Aplicadas (CIICAp)-Universidad Autónoma del Estado de Morelos (UAEM), Cuernavaca, Morelos, Mexico. ${ }^{3}$ Departmento de Ciencias de la Salud-Universidad de Sonora, Campus Cajeme, Sonora, Mexico. ${ }^{4}$ Laboratorio Multidisciplinario y Sección de Estudios de Posgrado e Investigación, Escuela Superior de Medicina, Instituto Politécnico Nacional, Mexico City, Mexico.

Received: 17 November 2015 Accepted: 12 July 2016

Published online: 21 July 2016

\section{References}

1. Barnes SK, Ozanne SE. Pathways linking the early environment to long-term health and lifespan. Prog Biophys Mol Biol. 2011;106(1):323-36.

2. Drake AJ, Reynolds RM. Impact of maternal obesity on offspring obesity and cardiometabolic disease risk. Reproduction. 2010;140(3):387-98.

3. Gaillard R. Maternal obesity during pregnancy and cardiovascular development and disease in the offspring. Eur J Epidemiol. 2015;30(11):1141-52.

4. Nicholas LM, Morrison JL, Rattanatray L, Zhang S, Ozanne SE, McMillen IC. The early origins of obesity and insulin resistance: timing, programming and mechanisms. Int J Obes (Lond). 2016:40(2):229-38. Epub 2015 Sep 14.

5. Poston L. Developmental programming and diabetes - The human experience and insight from animal models. Best Pract Res Clin Endocrinol Metab. 2010;24(4):541-52.

6. Remmers F, Delemarre-van de Waal HA. Developmental programming of energy balance and its hypothalamic regulation. Endocr Rev. 2011;32(2):272-311.

7. Symonds ME, Sebert SP, Hyatt MA, Budge H. Nutritional programming of the metabolic syndrome. Nat Rev Endocrinol. 2009;5(11):604-10.

8. Considine RV, Caro JF. Leptin: genes, concepts and clinical perspective. Horm Res. 1996;46(6):249-56.

9. Hassink SG, Sheslow DV, de Lancey E, Opentanova I, Considine RV, Caro JF. Serum leptin in children with obesity: relationship to gender and development. Pediatrics. 1996;98(2 Pt 1):201-3.

10. Zhang Y, Proenca R, Maffei M, Barone M, Leopold L, Friedman JM. Positional cloning of the mouse obese gene and its human homologue. Nature. 1994; 372(6505):425-32.

11. Koerner A, Kratzsch J, Kiess W. Adipocytokines: leptin-the classical, resistin-the controversical, adiponectin-the promising, and more to come. Best Pract Res Clin Endocrinol Metab. 2005;19(4):525-46. 
12. Konturek PC, Konturek JW, Czesnikiewicz-Guzik M, Brzozowski T, Sito E, Konturek SJ. Neuro-hormonal control of food intake: basic mechanisms and clinical implications. J Physiol Pharmacol. 2005;56 Suppl 6:5-25.

13. Lawlor DA, Relton C, Sattar N, Nelson SM. Maternal adiposity-a determinant of perinatal and offspring outcomes? Nat Rev Endocrinol. 2012;8(11):679-88.

14. Saladin R, De Vos P, Guerre-Millo M, Leturque A, Girard J, Staels B, Auwerx J. Transient increase in obese gene expression after food intake or insulin administration. Nature. 1995;377(6549):527-9.

15. Spiegelman BM, Flier JS. Obesity and the regulation of energy balance. Cell. 2001;104(4):531-43.

16. Morton GJ, Schwartz MW. Leptin and the central nervous system control of glucose metabolism. Physiol Rev. 2011;91(2):389-411.

17. Hoggard N, Hunter L, Duncan JS, Williams LM, Trayhurn P, Mercer JG. Leptin and leptin receptor mRNA and protein expression in the murine fetus and placenta. Proc Natl Acad Sci U S A. 1997;94(20):11073-8.

18. Shekhawat PS, Garland JS, Shivpuri C, Mick GJ, Sasidharan P, Pelz CJ, McCormick KL. Neonatal cord blood leptin: its relationship to birth weight, body mass index, maternal diabetes, and steroids. Pediatr Res. 1998;43(3):338-43.

19. Chiesa C, Osborn JF, Haass C, Natale F, Spinelli M, Scapillati E, Spinelli A, Pacifico L. Ghrelin, leptin, IGF-1, IGFBP-3, and insulin concentrations at birth: is there a relationship with fetal growth and neonatal anthropometry? Clin Chem. 2008;54(3):550-8.

20. Christou H, Connors JM, Ziotopoulou M, Hatzidakis V, Papathanassoglou E, Ringer SA, Mantzoros CS. Cord blood leptin and insulin-like growth factor levels are independent predictors of fetal growth. J Clin Endocrinol Metab. 2001;86(2):935-8.

21. Wolf HJ, Ebenbichler CF, Huter O, Bodner J, Lechleitner M, Foger B, Patsch $J R$, Desoye G. Fetal leptin and insulin levels only correlate inlarge-forgestational age infants. Eur J Endocrinol. 2000;142(6):623-9.

22. Boeke CE, Mantzoros CS, Hughes MD, Rifas-Shiman LS, Villamor E, Zera CA, Gillman MW. Differential associations of leptin with adiposity across early childhood. Obesity (Silver Spring). 2013;21(7):1430-7.

23. Mantzoros CS, Rifas-Shiman SL, Williams CJ, Fargnoli JL, Kelesidis T, Gillman MW. Cord blood leptin and adiponectin as predictors of adiposity in children at 3 years of age: a prospective cohort study. Pediatrics. 2009; 123(2):682-9.

24. Ong KK, Ahmed ML, Sherriff A, Woods KA, Watts A, Golding J, Dunger DB. Cord blood leptin is associated with size at birth and predicts infancy weight gain in humans. ALSPAC Study Team. Avon Longitudinal Study of Pregnancy and Childhood. J Clin Endocrinol Metab. 1999;84(3):1145-8.

25. Parker M, Rifas-Shiman SL, Belfort MB, Taveras EM, Oken E, Mantzoros C, Gillman MW. Gestational glucose tolerance and cord blood leptin levels predict slower weight gain in early infancy. J Pediatr. 2011;158(2):227-33.

26. Brunner S, Schmid D, Huttinger K, Much D, Heimberg E, Sedlmeier EM, Bruderl M, Kratzsch J, Bader BL, Amann-Gassner U, et al. Maternal insulin resistance, triglycerides and cord blood insulin in relation to post-natal weight trajectories and body composition in the offspring up to 2 years. Diabet Med. 2013;30(12):1500-7.

27. Rumelhart D. Feature discovering by competitive learning. Cogn Sci. 1985;9: 75-112.

28. Bishop. Neural networks and their applications. Rev Sci Instrument. 1994 65(6):1803-32

29. McCulloch WW, Pitts W. A logical calculus of ideas imminent in nervous activity. Bull Math Biophys. 1943;5:115-33.

30. Grossi E. Technology transfer from the science of medicine to the real world: the potential role played by artificial adaptive systems. Subst Use Misuse. 2007:42(2-3):267-304.

31. Grossi E, Buscema M. Artificial intelligence and outcome research. Drug Dev Res. 2006;67(3):227-44.

32. Grossi E, Buscema M. Introduction to artificial neural networks. Eur J Gastroenterol Hepatol. 2007;19(12):1046-54.

33. Chan $\mathrm{CH}$, Chan EY, Ng DK, Chow PY, Kwok KL. Application of artificial neural networks to establish a predictive mortality risk model in children admitted to a paediatric intensive care unit. Singapore Med J. 2006;47(11):928-34.

34. Patel $J$, Goyal RK. Applications of artificial neural networks in medical science. Curr Clin Pharmacol. 2007;2(3):217-26.

35. Street ME, Buscema M, Smerieri A, Montanini L, Grossi E. Artificial Neural Networks, and Evolutionary Algorithms as a systems biology approach to a data-base on fetal growth restriction. Prog Biophys Mol Biol. 2013;113(3):433-8.

36. Street ME, Grossi E, Volta C, Faleschini E, Bernasconi S. Placental determinants of fetal growth: identification of key factors in the insulin-like growth factor and cytokine systems using artificial neural networks. BMC Pediatr. 2008:8:24

37. Chowdhury DR, Chatterjee M, Samanta RK. An Artificial Neural Network model for neonatal disease diagnosis. Int J Artif Intell Expert Syst. 2011;2(3):96-106.

38. Karlik B, Vehbi A. Performance Analysis of Various Activation Functions in Generalized MLP Architectures of Neural Networks. Int J Artif Intell Expert Syst. 2011;1(4):111-22

39. Verma SP, Andaverde J, Santoyo E. Application of the error propagation theory in estimates of static formation temperatures in geothermal and petroleum boreholes. Energy Convers Manag. 2006;47:3659-71.

40. Demuth H, Beale M. Neural Network Toolbox for Matlab-User's guide version 3. M.A: Mathworks; 1998.

41. Heaton J. Introduction to the Math of Neural Networks. Heaton Res. 2016.

42. Rumelhart D, Hinton GE, Williams CJ. Learning representations by backpropagating errors. Nature. 1986;323:533-6.

43. Rumelhart DE, Hinton GE, Williams RJ. Learning internal representations by error propagation. Parallel Data Process. 1986;1:318-62.

44. Hagan MT, Menhaj MB. Training Feedforward Networks with the Marquardt Algorithm. IEEE Trans Neural Network. 1994;5(6):989-93.

45. Yu H, Wilamowski BM. Levenberg-Marquardt Training. In: Industrial electronics Handbook. Volume 5, edn. Florida, USA: CRC Press; 2011. 12-11 to 12-15.

46. Despange F, Massart DL. Neural networks in multivariate calibration. Analyst. 1998;123:157-78.

47. Verma SP, Andaverde J, Santoyo E. Application of the error propagation theory in estimates of static formation temperatures in geothermal and petroleum boreholes, Heat Transfer in Components and Systems for Sustainable Energy Technologies: Heat-SET 2005. In: Heat SET 2005: 2005; Grenoble, France: Proceeding from a Congress; 2005.

48. Uebel K, Pusch K, Gedrich K, Schneider KT, Hauner H, Bader BL. Effect of maternal obesity with and without gestational diabetes on offspring subcutaneous and preperitoneal adipose tissue development from birth up to year-1. BMC Pregnancy Childbirth. 2014;14:138.

49. Ben X, Qin Y, Wu S, Zhang W, Cai W. Placental leptin correlates with intrauterine fetal growth and development. Chin Med J (Engl). 2001;114(6):636-9.

50. Gross GA, Solenberger T, Philpott T, Holcomb Jr WL, Landt M. Plasma leptin concentrations in newborns of diabetic and nondiabetic mothers. Am J Perinatol. 1998;15(4):243-7.

51. Persson B, Westgren M, Celsi G, Nord E, Ortqvist E. Leptin concentrations in cord blood in normal newborn infants and offspring of diabetic mothers. Horm Metab Res. 1999;31(8):467-71.

52. Aleboyeh A, Kasiri MB, Olya ME, Aleboyeh $\mathrm{H}$. Prediction of azo dye decolorization by UV/H2O2 using artificial neural networks. Dyes Pigments. 2008;77:288-94.

53. Garson GD. Interpreting neural-network connection weights. Al Experts. 1991;6:47-51.

54. Koistinen HA, Koivisto VA, Andersson S, Karonen SL, Kontula K, Oksanen L, Teramo KA. Leptin concentration in cord blood correlates with intrauterine growth. J Clin Endocrinol Metab. 1997:82(10):3328-30.

55. Rafeey M, Ouladsahebmadarek E, Rashtchizadeh N, Sheikh Monazah F, Gorbanihaghjo A, Hosseini MB, Nejati N. Correlation between maternal and cord blood leptin and fetal growth. Afr J Biotechnol. 2007;6(17):2013-27.

56. Denison FC, Roberts KA, Barr SM, Norman JE. Obesity, pregnancy, inflammation, and vascular function. Reproduction. 2010;140(3):373-85.

57. Leperca J, Cauzac M, Lahlou N, Timsit J, Girard J, Auwerx J, Hauguel-de Mouzon S. Overexpression of placental leptin in diabetic pregnancy: a critical role for insulin. Diabetes. 1998;47(5):847-50

58. Fietta P. Focus on leptin, a pleiotropic hormone. Minerva Med. 2005;96(2):65-75.

59. Kaar JL, Brinton JT, Crume T, Hamman RF, Glueck DH, Dabelea D. Leptin levels at birth and infant growth: the EPOCH study. J Dev Orig Health Dis. 2014;5(3):214-8

60. Boskovic R, Feig DS, Derewlany L, Knie B, Portnoi G, Koren G. Transfer of insulin lispro across the human placenta: in vitro perfusion studies. Diabetes Care. 2003;26(5):1390-4.

61. Catalano PM, McIntyre HD, Cruickshank JK, McCance DR, Dyer AR, Metzger BE, Lowe LP, Trimble ER, Coustan DR, Hadden DR, et al. The hyperglycemia and adverse pregnancy outcome study: associations of GDM and obesity with pregnancy outcomes. Diabetes Care. 2012;35(4):780-6. 